\title{
Forkhead box Q1: A key player in the pathogenesis of tumors (Review)
}

\author{
YANG LI ${ }^{1}$, YEFEI ZHANG ${ }^{1}$, ZHENDONG YAO $^{1}$, SISI LI $^{2}$, ZHENHUA YIN $^{2}$ and MIN XU ${ }^{1,2}$ \\ ${ }^{1}$ Department of Gastroenterology, Shanghai General Hospital of Nanjing Medical University; \\ ${ }^{2}$ Department of Gastroenterology, Shanghai General Hospital of \\ Shanghai Jiaotong University, Hongkou, Shanghai 200080, P.R. China
}

Received February 21, 2016; Accepted March 30, 2016

DOI: $10.3892 /$ ijo.2016.3517

\begin{abstract}
As a member of the Forkhead box protein family, Forkhead box Q1 (FOXQ1) is a transcription factor that functions to regulate cell differentiation. Recently, an increasing number of studies have demonstrated that FOXQ1 is significantly associated with the pathogenesis of tumors. This review aims to predominantly discuss the relationship between FOXQ1 and various types of tumor. The FOXQ1 gene is located at human chromosome 6p25.3 and encodes a functional 403 amino acid protein, which has many physiological functions, including promoting epithelial differentiation, inhibiting smooth muscle differentiation, activating $\mathrm{T}$ cells and autoimmunity, and controlling mucin gene expression and granule content in stomach surface mucous cells. There are several modes of regulation of FOXQ1 expression that have been demonstrated in normal and tumor cells, such as microRNA and the Wnt signaling pathway. The activation of FOXQ1 affects downstream genes promoting the initiation, proliferation and invasion, in addition to the metastasis of tumor cells. Amongst these, the regulation of invasion and metastasis by FOXQ1 is the most extensively studied. The detailed mechanism involves angiogenesis, tumor re-initiation, alterations in the tumor microenvironment and epithelial-mesenchymal transition. In a number of studies, the expression of FOXQ1 has been reported to be upregulated in breast, colorectal, pancreatic, bladder and ovarian cancer, and glioma, amongst other tumor types. Together, these studies contribute to cancer diagnostics, prognostics and therapeutics. In conclusion, the application prospect of FOXQ1 in tumors is hopeful.
\end{abstract}

Correspondence to: Professor Min Xu, Department of Gastroenterology, Shanghai General Hospital of Nanjing Medical University, 100 Haining Road, Hongkou, Shanghai 200080, P.R. China E-mail: xuminmd@163.com

Key words: FoxQ1, forkhead box, tumor, transcription factor, invasion, metastasis

\section{Contents}

1. Introduction

2. FOXQ1 structure and physiological function

3. The regulation of FOXQ1 expression

4. FOXQ1 and tumor biology

5. Clinical significance of FOXQ1 expression

6. FOXQ1 and tumor therapy

7. Conclusions

\section{Introduction}

The Forkhead box (FOX) transcription factor was a mutant that was first identified in Drosophila melanogaster in 1989, and was initially called Drosophila homeotic forkhead (fkh) protein (1). At that time, all that was known was that FOX was a nuclear protein and was associated with transcriptional regulation; however, little was known regarding the detailed structure and function. The subsequent discovery of the rat gene, hepatocyte nuclear factor-3 (HNF-3), brought to light a previously unknown family of transcription factors carrying the 'Forkhead' motif (2). Since then, studies have increased regarding the presence of FOX transcription factors (3-5).

Currently, the FOX transcription factor family is known to consist of $>100$ members, which are classified into 19 subfamilies, called FOXA-S $(2,6)$. The 19 subfamilies are involved in cell differentiation, proliferation and apoptosis, embryonic development, ageing, glucose and lipid metabolism, and immune regulation, which serves an important role in human health and disease, particularly in cancer (7). Recently, a number of studies have demonstrated that FOX transcription factors are associated with the initiation, progression and metastasis of human cancers (8-12). As a member of the FOX transcription factor family, FOXQ1 is a major oncogenic transcription factor, similar to other subsets of FOX transcription factors, including FOXA1, FOXC1, FOXC2, FOXG1, FOXM1 and FOXO. Several lines of evidence reported in the literature demonstrate a key role for FOXQ1 in the progression of tumors (13-15). In this review, we discuss the FOXQ1 structure and the association between FOXQ1 and tumors, in addition to the application prospect of FOXQ1 in tumors. 


\section{FOXQ1 structure and physiological function}

The FOXQ1 gene, in addition to the FOXF2 and FOXC1 genes, is located at human chromosome 6p25.3 and consists of 2319 base pairs (bp) (16). FOXQ1 is encoded by an open reading frame of $1029 \mathrm{bp}$, producing a functional 403 amino acid protein. The FOXQ1 transcription factor, also called HNF-3/fkh homolog-1 (HFH-1), is a DNA-binding protein that regulates the cell cycle. The FOXQ1 protein is divided into three domains: The alanine and glycine enrichment region, the forkhead box domain (FHD) containing 96-amino acids and the proline-enrichment region. The FHD, also known as the Winged-helix domain, is the DNA binding domain, while the other two are associated with transcriptional activators, transcriptional repressors or DNA repair complexes. The core of this polypeptide contains three $\alpha$-helices $(\alpha 1, \alpha 2$ and $\alpha 3)$ and two wing-like loops located side by side. Among these three helices, $\alpha 3$ is the most significant part as it contains the DNA binding sequence (7). In 1994, Overdier et al (3) reported a core FOXQ1 binding sequence of 5'-TGTTTA-3'. However, more recently, Abba et al (17) suggested that the precise binding sequence may be 5'-GTTT-3'. By searching Transfac (www.biobase-international.com) and the JASPER CORE database (www.jasper.cgb.ki.se), matrix motifs were identified in rat alone, with a core 'GTTT' motif. Compared with other genes, FOXQ1 is evolutionarily conserved, and the forkhead DNA binding domain of the human, mouse and rat FOXQ1 proteins share $100 \%$ sequence identity, suggesting that the 5'-GTTT-3' core may be valid across all species (Fig. 1) (17).

FOXQ1 was highly expressed in the mouse stomach, as reported by Bieller et al (18). In this study, the authors measured FOXQ1 expression levels in human gastric mucosa and muscle, which indicated that the transcripts were present in the gastric mucosa tissue but not in the gastric muscle tissue. Additionally, the authors investigated human FOXQ1 expression in different tissues, observing strong expression of FOXQ1 in the stomach, trachea, bladder and salivary glands, and significant expression in the duodenum, prostate and fetal liver tissue (18).

There are numerous physiological functions of FOXQ1, which have been reported in previous studies, including promoting epithelial differentiation, inhibiting smooth muscle differentiation, activating $\mathrm{T}$ cells and autoimmunity, controlling mucin gene expression and granule content in stomach surface mucous cells (19-21). Additionally, FOXQ1 has been demonstrated to regulate hair differentiation. Hong et al (22) reported that the hair of the satin mouse could been modified by FOXQ1. Further study indicated that a key role of FOXQ1 was as a transcription factor and target of homeobox $\mathrm{C} 13$ regulation; a member of the Homeobox gene family that is able to control hair follicle development and hair growth $(23,24)$. Future investigations of FOXQ1 will provide valuable insight into its physiological function.

\section{The regulation of FOXQ1 expression}

There are several modes that have been demonstrated to regulate FOXQ1 expression in normal and tumor cells. Herein, we present some modes of FOXQ1 regulation in human health and disease, focusing on the modes of regulation of FOXQ1 in tumors in particular (Fig. 2).

The relationship between microRNA (miRNA or miR) and FOXQ1. miRNAs are small noncoding regulator RNAs comprised of 18-25 nucleotides, which are able to control the translation of mRNA involved in cellular processes, such as cell proliferation and apoptosis (25-27). miRNAs combine with other proteins to form the RNA-induced silencing complex (RISC), which is able to bind to the 3'-untranslated region (3'UTR) of a target mRNA, regulating the translation of the target gene (28). A number of studies have directly or indirectly indicated that miRNAs are associated with types of human cancer, including breast, colorectal and pancreatic cancer (29-31). Recently, studies have demonstrated that several miRNAs function as tumor regulators by targeting FOXQ1. In this review, we describe some of these miRNAs.

Peng et al (32) demonstrated that miR-124 was able to inhibit the proliferation, migration and invasion of nasopharyngeal carcinoma cells through directly targeting the FOXQ1 3'-UTR. Using reverse transcription-quantitative polymerase chain reaction (RT-qPCR), it was observed that FOXQ1 is highly conserved among different species, with the 3'-UTR of the mRNA containing a complementary site for the seed region of miR-124. Subsequently, dual-luciferase reporter vectors containing the target region sequence of FOXQ1 3'-UTR (wt3'-UTR) or the mutant sequence (mut 3'-UTR) were generated. The results revealed that miR-124 was able to downregulate the luciferase activity of the FOXQ1 wt3'-UTR construct, but not the mut 3'-UTR, suggesting that FOXQ1 is a direct target of miR-124. Additionally, a rescue experiment indicated that the overexpression of FOXQ1 could partially rescue the suppression of miR-124. Similarly, miR-422a, miR-506 and miR-1271 have been found to regulate the expression of FOXQ1 (33-35).

Oncogenic signaling pathways activate FOXQ1 in cancer cells. Dysfunction in the signaling pathway may lead to the loss of cell cycle control, and as a result, common epithelial cells transform into cancer cells, and normal tissue become neoplastic (36). Consistent with this, studies have reported that the expression of FOXQ1 is mediated by certain signaling pathways.

The pathway predominantly involved is the $\mathrm{Wnt} / \beta$-catenin signaling pathway, which was first described by Nusse and Varmus in 1982 (37). Increasingly, studies have reported the functions of the Wnt/ $\beta$-catenin signaling pathway, particularly its role in the initiation, progression and metastasis of human carcinoma (38). Additionally,FOXQ1 is a downstream mediator in the Wnt/B-catenin signaling pathway. Christensen et al (39) reported that the upregulation of FOXQ1 led to the loss of the expression of caudal-related homeodomain transcription 2, a transcription factor associated with Wnt signaling pathway activity. In addition, glycogen synthase kinase 3, a small molecular inhibitor typical of the Wnt pathway, was demonstrated to result in increased levels of FOXQ1 mRNA and protein, similar to a constitutively active form of $\beta$ catenin. Furthermore, the Wnt pathway was demonstrated to regulate FOXQ1 expression via the identified transcription factor-4 binding site (39). 

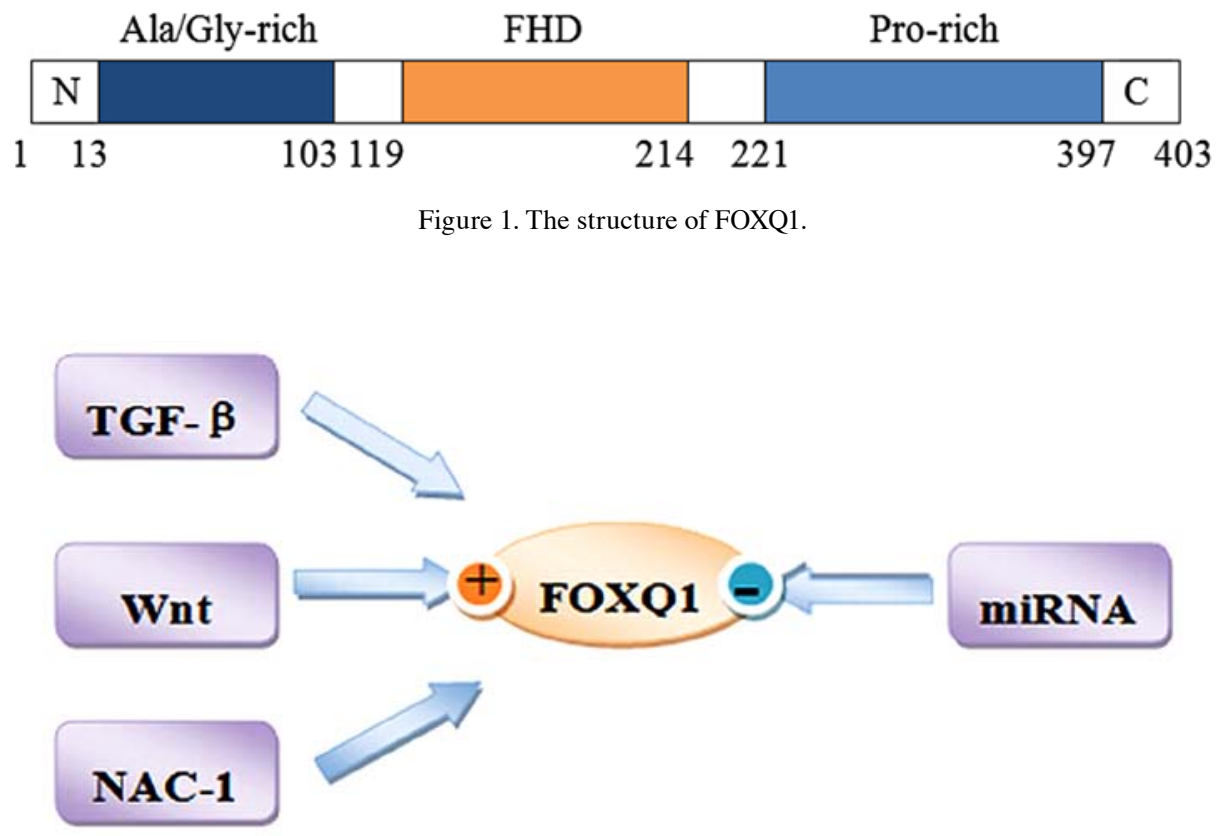

Figure 2. The regulation of FOXQ1 expression.

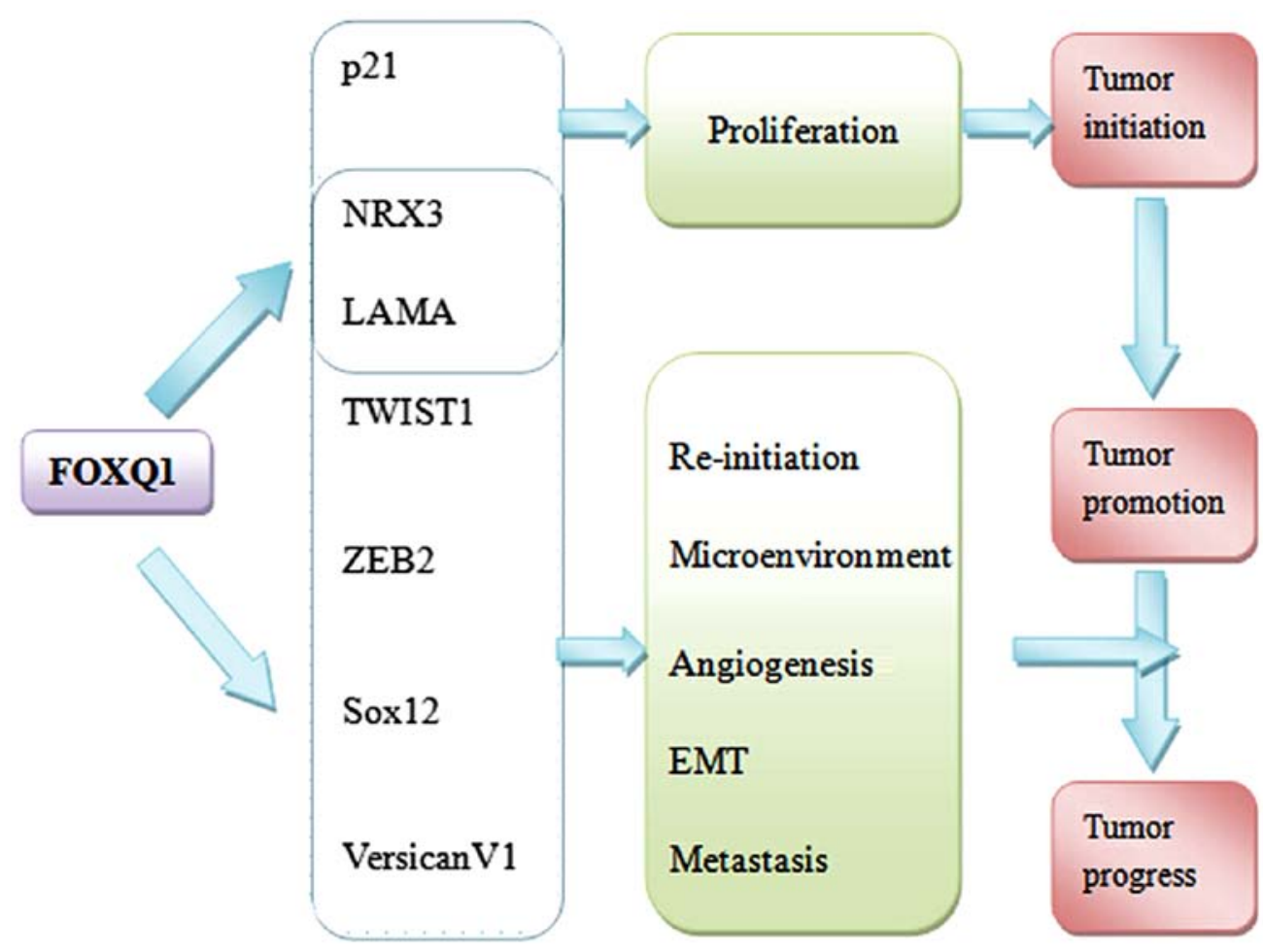

Figure 3. Function of FOXQ1 in cancer cells.

Similarly, it has been reported that FOXQ1 is upregulated by the transforming growth factor (TGF) $\beta$ signaling pathway (40). The TGF- $\beta$ /mothers against decapentaplegic (SMAD) signaling pathway has been demonstrated to regulate expression in epithelial-mesenchymal transition (EMT) (41). Although the underlying mechanisms are unknown, treating cancer cells with TGF- $\beta$ for 3 days markedly increased FOXQ1 mRNA and protein levels. Interestingly, further investigation of these cells indicated alterations in the Wnt pathway, including vascular endothelial growth factor (VEGF)-A, matrix metalloproteinase 2, vimentin, N-cadherin and E-cadherin, from which it may be inferred that FOXQ1 provides crosstalk between the Wnt and TGF- $\beta$ signaling pathways (42).

Nucleus accumbens-associated protein 1 (NACl) regulates FOXQ1 in cancer cells. NAC1 is a transcriptional coregulator, which participates in various biological processes. For instance, it is NACl that regulates bony patterning in the 
murine vertebral column, is involved in acute psychomotor stimulant responses and contributes to tumor progression, tumor cell proliferation and survival (43-46). Gao et al (47) reported that FOXQ1 expression is transcriptionally regulated by NAC1. In addition, microarray analysis of gene expression and RT-qPCR indicated that Notch1 and Jagged1, two important factors in the Notch signaling pathway, were also regulated by NAC1. However, whether FOXQ1 is associated with the Notch signaling pathway remains unknown (47).

\section{FOXQ1 and tumor biology}

Tumor biology is a complex process involving cancer initiation, proliferation and invasion, in addition to metastasis. It is known that sustaining proliferative signaling, evading growth suppressors, resisting cell death, limitless replicative immortality and deregulated cellular energetics supports tumorigenesis (48). Several studies have reported that numerous genes downstream of FOXQ1 function at every stage of tumorigenesis (Table I and Fig. 3).

Roles of FOXQ1 expression in tumor initiation and proliferation. FOXQ1 belongs to the FOX gene family, which is involved in the regulation of the cell cycle (49). For example, FOXM1 is known to modify the G1/S and G2/M transitions and M-phase progression in the cell cycle (50). Similarly, FOXQ1 was able to promote epithelial differentiation and inhibit smooth muscle differentiation. However, with FOXQ1 overexpression, tumorigenesis is uncontrolled (51). Knockdown of FOXQ1 partially mimics miR-422a function, suppressing tumorigenesis in hepatocellular carcinoma (HCC) cells in vitro. In addition, tumor volume and weight in FOXQ1-knockdown mice were significantly increased. Thus, FOXQ1 serves a critical role in tumor initiation (33).

FOXQ1 may promote tumor cell proliferation, while the downregulation of FOXQ1 inhibits proliferation. Compared with the involvement of FOXQ1 in tumor initiation, studies have demonstrated that it may promote tumor cell proliferation by upregulating downstream genes encoding proliferationassociated proteins and downregulating apoptotic proteins.

Neurexin (NRXN) is a polymorphic neuronal-specific cell surface protein, which serves an important role in cell adhesion and recognition (52). It is encoded by three genes, NRXN1, NRXN2 and NRXN3, which together form two functional proteins; the long form, $\alpha-N R X N$, and the short form, $\beta$-NRXN (52-54). It has been reported that a polymorphic site in the NRXN3 gene was significantly associated with a higher risk of developing breast cancer (55). Recently, Sun et al (56) observed that FOXQ1 is overexpressed in human glioma, and is negatively correlated with NRXN3 expression in clinical tissue. Additionally, FOXQ1 was observed to regulate the activity of NRXN3, which enhanced cell proliferation. To further understand the associated mechanism, three mutant versions of the FOXQ1-binding sites in NRXN3 were generated: A FOXQ1-binding site 1 mutation only, a FoxQ1-binding site 2 mutation only and both site 1 and site 2 mutations. These demonstrated that disruption of one or both of the FOXQ1 binding sites significantly inhibited the NRXN3 promoter. Consequently, NRXN3 is a downstream gene regulated by FOXQ1, which can promote cancer proliferation.
Table I. Genes targeted by FOXQ1 in human tumor progress.

\begin{tabular}{lll}
\hline Tumor type & \multicolumn{1}{c}{ Target gene } & Refs. \\
\hline Glioma & NRX3 & $(56)$ \\
Colorectal cancer & p21 & $(60)$ \\
HCC & ZEB2, VersicanV1 & $(74)$ \\
& SOX12 & $(87)$ \\
Breast cancer & TWIST1 & $(51)$ \\
& LAMA4 & $(69)$ \\
\hline
\end{tabular}

p21 is a cyclin-dependent kinase inhibitor, which functions as a regulator of cell cycle progression (57). A number of studies have demonstrated the negative correlation between polymorphisms in p21 and the susceptibility of certain malignant tumors $(58,59)$. Recently, at the molecular level, a large body of literature suggests that $\mathrm{p} 21$ has negative roles in tumor growth using FOXQ1-overexpressing cells in which p21 is knocked down. In addition, p21 induction by FOXQ1 is p53 independent. By contrast, FOXQ1 overexpression did not affect p21 in vivo, and the pathogenesis of FOXQ1 in mediating p21 change to control tumor antiapoptosis requires further in-depth study $(17,60)$.

Roles of FOXQ1 expression in tumor angiogenesis. Sustained angiogenesis is a key step in cancer development and involves the development of new blood vessels necessary for continued tumor growth and metastatic spread. Increasingly, studies have shown that angiogenic regulators and the activation of oncogenes are essential for the maintenance of an angiogenic phenotype that contributes to oncogenesis (61-63). Studies have shown that FOXQ1 is a protein that promotes tumor angiogenesis (42). Christensen et al (39) observed that knockdown of FOXQ1 expression suppressed the angiogenic capacity of SW480 colorectal cancer cells via the regulation of VEGF, which is an activator of angiogenesis that is secreted by tumor cells (64). Microarray analysis revealed that VEGF-A expression was upregulated 4.4-fold, suggesting the possibility of enhanced angiogenesis. RT-qPCR in SW480 cells and VEGF staining of tumor specimens confirmed this result. Despite this, the detailed mechanisms of FOXQ1-mediated angiogenesis, for example whether FOXQ1 protein is able to bind to the 3'UTR of the VEGF gene, requires further study.

Roles of FOXQ1 expression in tumor re-initiation. Tumor re-initiation, which assesses the capacity of human cancer cells to proliferate once implanted into a secondary host, is another characteristic of malignant tumors $(65,66)$. Laminin $\alpha 4$, encoded by LAMA4, is a major component of the extracellular matrix, and has been implicated in cancer pathophysiology $(67,68)$. In a recent study, FOXQ1 was observed to positively regulate the expression of LAMA4, which promoted the development of micrometastasis and tumor re-initiation in vivo (69).

Roles of FOXQ1 expression in tumor microenvironment and tumor promotion inflammation. Pathologists have long recognized that inflammation can supply bioactive molecules 
to the tumor microenvironment, including growth factors that sustain proliferative signaling and survival factors that limit cell death, thereby promoting cell proliferation, EMT, invasion and metastasis (70-72). Of note, FOXQ1 alters the tumor microenvironment through regulating versican V1. Researchers confirmed that versican $\mathrm{V} 1$, which promoted the metastasis of HCC cells and promotes the recruitment of macrophages, is a direct transcriptional target of FOXQ1. Versican V1 overexpression regulated FOXQ1 and induced HCC cells to secrete chemokine ligand 2 (CCL2), which was able to increase tumorassociated macrophages, whereas inhibiting versican V1 can significantly inhibit FOXQ1 expression. In addition, RT-qPCR assay indicated that the levels of CCL2, TNF- $\alpha$, IL-6 and IL-8, all of which are implicated in macrophage recruitment and inflammation, were significantly upregulated in versican V1 overexpressing HCC tissues compared with the control HCC tissues $(73,74)$.

Roles of FOXQ1 expression in tumor EMT. The transition of epithelial cells to mesenchymal cells in EMT, is an indicator of tumor metastasis (75). Epithelial cells are surface barrier cells, while mesenchymal cells have scaffolding and anchoring functions, and roles in tissue repair and wound healing $(76,77)$. In the EMT process regulated by FOXQ1, mesenchymal cell markers, such as E-cadherin (E-cad), increase while epithelial cell markers, such as vimentin (VIM), are reduced. EMT-associated protein expression in the non-small cell lung cancer cell lines, SPC-A-1-SCR and NCI-H1395-SCR, indicated that the levels of E-cad and mucoprotein increase, whilst VIM and calcium-binding protein S100A4 expression is reduced (78). Similar results were observed in studies of bladder, ovarian and breast cancer cells (79-81).

Furthermore, an increasing number of studies have reported two tumor-specific patterns of regulating the downstream genes of FOXQ1, which contribute to the EMT process in tumors. A chromatin immunoprecipitation assay in breast cancer cells indicated that FOXQ1 is able to bind to the promoter of E-cad directly $(81,82)$, while FOXQ1 enhanced genes in other tumors indirectly, such as Twist-related protein 1 (TWIST1) in colon cancer cells and NRXN3 in glioma $(17,56)$.

Roles of FOXQ1 expression in tumor invasion and metastasis. Tumor metastasis is a complex multistep process including the following steps: Invasion of primary tumor cells, new blood or lymphatic vessel formation, transportation of tumor cells via the blood or lymphatic vessels, and the re-initiation of tumor cells at the secondary site (83). Metastasis serves a crucial role in the morbidity and mortality of cancer. Although studies regarding the capability of invasion and metastasis have increased over the past decade, the molecular mechanisms which control these steps remain poorly understood $(84,85)$.

As powerful new research tools and refined experimental methods have become available, studies have identified FOXQ1 as one of the critical regulators in tumor invasion and metastasis. Recent studies have suggested that FOXQ1 is overexpressed in human tumor tissues and is associated with the incidence of tumor metastasis and poor tumor TNM stage. Additionally, detailed experiments found that FOXQ1 could mediate all steps of tumor metastasis, from the initial EMT to the ultimate organotropic colonization. To be a critical
Table II. Expression of FOXQ1 and its clinical significance.

\begin{tabular}{|c|c|c|c|c|c|}
\hline \multirow[b]{2}{*}{ Tumor type } & \multicolumn{2}{|c|}{$\begin{array}{l}\text { Expression } \\
\text { of FOXQ1 }\end{array}$} & \multirow[b]{2}{*}{$\begin{array}{l}\text { TNM } \\
\text { stage }\end{array}$} & \multirow{2}{*}{$\begin{array}{c}\text { Independent } \\
\text { prognostic } \\
\text { factor }\end{array}$} & \multirow[b]{2}{*}{ Refs } \\
\hline & Cell & $\begin{array}{l}\text { Tumor } \\
\text { tissue }\end{array}$ & & & \\
\hline Breast cancer & Up & & & & (97) \\
\hline Colorectal cancer & $\mathrm{Up}$ & Up & Yes & No & (60) \\
\hline Gastric cancer & $\mathrm{Up}$ & Up & Yes & & (98) \\
\hline Esophagus cancer & $\mathrm{Up}$ & Up & & & (86) \\
\hline $\mathrm{HCC}$ & Up & $\mathrm{Up}$ & Yes & Yes & (99) \\
\hline NSCLC & $\mathrm{Up}$ & $\mathrm{Up}$ & Yes & Yes & (100) \\
\hline Pancreatic cancer & $\mathrm{Up}$ & $\mathrm{Up}$ & Yes & Yes & (101) \\
\hline $\mathrm{NPC}$ & Up & Up & Yes & & $(32)$ \\
\hline Bladder cancer & Up & Up & & & (79) \\
\hline Glioma & Up & Up & & & (56) \\
\hline Cervical cancer & Up & & & & (40) \\
\hline Ovarian cancer & Up & & & & (47) \\
\hline
\end{tabular}

regulator of tumor invasion and metastasis, studies have demonstrated that FOXQ1 promotes these steps by regulating the expression of downstream genes, such as zinc finger E-box binding homeobox (ZEB2), TWIST1 and Sry (sex determining region Y)-box 12 (SOX12) $(74,86,87)$.

ZEB2, encoded by the ZEB2 gene, promotes tumor metastasis (88-90). Recently, it was identified that ZEB2 is essential for FOXQ1-mediated HCC metastasis, with FOXQ1 transactivating ZEB2 expression by directly binding to the ZEB2 promoter. Xia et al (74) reported that the downregulation of ZEB2 in HCC cells significantly reduced the capacity for FOXQ1-enhanced cell metastasis, whereas the upregulation of ZEB2 rescued the reduced metastatic abilities. Subsequently, a PCR array indicated that, when induced by FOXQ1 knockdown, the ZEB2 mRNA expression profiles of SMMC7721-FOXQ1 cells were upregulated 4.38-fold compared with the profiles of SMMC7721-control cells. Additionally, a FOXQ1-binding site was constructed in the ZEB2 promoter, which demonstrated that the FOXQ1 protein directly bound to it. However, in a similar experiment using colorectal cancer cells, opposing results were observed, with nearly all proteins associated with the Wnt signaling pathway unaltered, revealing that the underlying mechanisms remain unknown.

SOX12, at human chromosome 20p13, belongs to the SYR-related high mobility group box (SOX) family. SOX family proteins are critical for a number of physiological processes including the maintenance of stem cells and controlling terminal differentiation of a variety of cell types (91-93). SOX12 is enriched in human HCC tissues, in addition to the invasion and metastasis of HCC cells. Additionally, studies have indicated that SOX12 may transactivate TWIST1, which is able to directly bind to $\mathrm{CDH} 1$, which encodes E-cad, regulating cell migration (94). Fibroblast growth factor-binding protein 1 (FGFBP1) is a target of SOX12 and is secreted. Additionally, it has been shown to promote cancer growth, angiogenesis and metastasis $(95,96)$. 


\section{Clinical significance of FOXQ1 expression}

The identification of specific and sensitive biomarkers would improve the selection of tumor patients for individualized treatment. A number of in vitro and in vivo animal experiments have indicated a key role of FOXQ1 in the pathogenesis of various types of tumor. Additionally, studies have indicated that FOXQ1 is overexpressed in human tumor specimens and may be an indicator of poor prognosis (Table II). Therefore, FOXQ1 may have potential for the diagnosis and treatment of tumors.

\section{FOXQ1 and tumor therapy}

FOXQ1 is a transcription factor that is overexpressed in a number of different types of cancer cells (97). An increasing number of studies have indicated that the upregulation of FOXQ1 promotes resistance to chemotherapeutic drugs $(42,51)$. However, the therapeutic potential of FOXQ1 inhibitors against tumor remains to be explored. The most efficient method is RNA interference, which has been widely investigated in chronic infectious arthritis, obesity and cancer $(98,99)$. Studies have demonstrated that the downregulation of FOXM1 expression by small interfering RNA diminished the proliferation and metastasis of numerous cells $(17,69,97,100)$. Following transfection of a short hairpin RNA (shRNA), eukaryotic expression vector (FOXQl-shRNA) targeting the human FOXQl gene, EMT of tumors reversed. Another compound is benzyl isothiocyanate (BITC) treatment which, prior to a carcinogen challenge, inhibited polycyclic aromatic hydrocarbon-induced mammary cancer in human (101). Anuradha et al (81) reported that the suppression of FOXQ1, at least in part, contributed to the BITC-mediated inhibition of cell migration.

\section{Conclusion}

FOXQ1 is closely associated with the occurrence of tumors, such as breast, non-small cell lung, colorectal and pancreatic cancer. Each step of tumorigenesis regulated by FOXQ1, including cell proliferation, invasion and apoptosis, has attracted significant research attention. Its function as a point of crosstalk between typical signaling pathways, including Wnt/ $\beta$-catenin, TGF- $\beta / S M A D$, Hedgehog and Notch, will provide valuable insight into the mechanisms of FOXQ1 in tumor pathophysiology. FOXQ1 is predicted to become a key marker of cancer diagnosis and therapy.

\section{References}

1. Weigel D, Jürgens G, Küttner F, Seifert E and Jäckle H: The homeotic gene fork head encodes a nuclear protein and is expressed in the terminal regions of the Drosophila embryo. Cell 57: 645-658, 1989.

2. Kaestner KH, Knochel W and Martinez DE: Unified nomenclature for the winged helix/forkhead transcription factors. Genes Dev 14: 142-146, 2000.

3. Overdier DG, Porcella A and Costa RH: The DNA-binding specificity of the hepatocyte nuclear factor $3 /$ forkhead domain is influenced by amino-acid residues adjacent to the recognition helix. Mol Cell Biol 14: 2755-2766, 1994.

4. Lai E, Prezioso VR, Smith E, Litvin O, Costa RH and Darnell JE Jr: HNF-3A, a hepatocyte-enriched transcription factor of novel structure is regulated transcriptionally. Genes Dev 4: 1427-1436, 1990.
5. Lai E, Prezioso VR, Tao WF, Chen WS and Darnell JE Jr: Hepatocyte nuclear factor 3 alpha belongs to a gene family in mammals that is homologous to the Drosophila homeotic gene fork head. Genes Dev 5: 416-427, 1991.

6. Benayoun BA, Caburet S and Veitia RA: Forkhead transcription factors: Key players in health and disease. Trends Genet 27: 224-232, 2011.

7. Carlsson P and Mahlapuu M: Forkhead transcription factors: Key players in development and metabolism. Dev Biol 250: 1-23, 2002.

8. Lin L, Miller CT, Contreras JI, Prescott MS, Dagenais SL, Wu R, Yee J, Orringer MB, Misek DE, Hanash SM, et al: The hepatocyte nuclear factor 3 alpha gene, HNF3alpha (FOXA1), on chromosome band 14q13 is amplified and overexpressed in esophageal and lung adenocarcinomas. Cancer Res 62: 5273-5279, 2002.

9. Li J and Vogt PK: The retroviral oncogene qin belongs to the transcription factor family that includes the homeotic gene fork head. Proc Natl Acad Sci USA 90: 4490-4494, 1993.

10. Koo CY, Muir KW and Lam EW: FOXM1: From cancer initiation to progression and treatment. Biochim Biophys Acta 1819: 28-37, 2012.

11. Nakamura T, Furukawa Y, Nakagawa H, Tsunoda T, Ohigashi H, Murata K, Ishikawa O, Ohgaki K, Kashimura N, Miyamoto M, et al: Genome-wide cDNA microarray analysis of gene expression profiles in pancreatic cancers using populations of tumor cells and normal ductal epithelial cells selected for purity by laser microdissection. Oncogene 23: 2385-2400, 2004.

12. Myatt SS and Lam EW: The emerging roles of forkhead box (Fox) proteins in cancer. Nat Rev Cancer 7: 847-859, 2007.

13. Cao D, Hustinx SR, Sui G, Bala P, Sato N, Martin S, Maitra A, Murphy KM, Cameron JL, Yeo CJ, et al: Identification of novel highly expressed genes in pancreatic ductal adenocarcinomas through a bioinformatics analysis of expressed sequence tags. Cancer Biol Ther 3: 1081-1089, discussion 1090-1091, 2004.

14. Tang H, Guo Q, Zhang C, Zhu J, Yang H, Zou YL, Yan Y, Hong D, Sou T and Yan XM: Identification of an intermediate signature that marks the initial phases of the colorectal adenomacarcinoma transition. Int J Mol Med 26: 631-641, 2010.

15. Feuerborn A, Srivastava PK, Küffer S, Grandy WA, Sijmonsma TP, Gretz N, Brors B and Gröne HJ: The Forkhead factor FoxQ1 influences epithelial differentiation. J Cell Physiol 226: 710-719, 2011

16. Katoh $\mathrm{M}$ and Katoh M: Human FOX gene family (Review). Int J Oncol 25: 1495-1500, 2004.

17. Abba M, Patil N, Rasheed K, Nelson LD, Mudduluru G, Leupold JH and Allgayer H: Unraveling the role of FOXQ1 in colorectal cancer metastasis. Mol Cancer Res 11: 1017-1028, 2013.

18. Bieller A, Pasche B, Frank S, Gläser B, Kunz J, Witt K and Zoll B: Isolation and characterization of the human forkhead gene FOXQ1. DNA Cell Biol 20: 555-561, 2001.

19. Hannenhalli S and Kaestner KH: The evolution of Fox genes and their role in development and disease. Nat Rev Genet 10: 233-240, 2009.

20. Hoggatt AM, Kriegel AM, Smith AF and Herring BP: Hepatocyte nuclear factor-3 homologue 1 (HFH-1) represses transcription of smooth muscle-specific genes. J Biol Chem 275: 31162-31170, 2000.

21. Jonsson H and Peng SL: Forkhead transcription factors in immunology. Cell Mol Life Sci 62: 397-409, 2005.

22. Hong H-K, Noveroske JK, Headon DJ, Liu T, Sy MS, Justice MJ and Chakravarti A: The winged helix/forkhead transcription factor Foxq1 regulates differentiation of hair in satin mice. Genesis 29: 163-171, 2001.

23. Potter CS, Peterson RL, Barth JL, Pruett ND, Jacobs DF, Kern MJ, Argraves WS, Sundberg JP and Awgulewitsch A: Evidence that the satin hair mutant gene Foxq1 is among multiple and functionally diverse regulatory targets for Hoxc13 during hair follicle differentiation. J Biol Chem 281: 29245-29255, 2006.

24. Wu B, Herbert Pratt C, Potter CS, Silva KA, Kennedy V and Sundberg JP: R164C mutation in FOXQ1 H3 domain affects formation of the hair medulla. Exp Dermatol 22: 234-236, 2013.

25. Calin GA and Croce CM: MicroRNA signatures in human cancers. Nat Rev Cancer 6: 857-866, 2006.

26. Garzon R, Fabbri M, Cimmino A, Calin GA and Croce CM: MicroRNA expression and function in cancer. Trends Mol Med 12: 580-587, 2006. 
27. Narasimhan K, Gauthaman K, Pushparaj PN, Meenakumari G, Chaudhary AGA, Abuzenadah A, Gari MA, Al Qahtani M and Manikandan J: Identification of unique miRNA biomarkers in colorectal adenoma and carcinoma using microarray: evaluation of their putative role in disease progression. ISRN Cell Biol 2014: 1-10, 2014. doi: org/10.1155/2014/526075.

28. Valencia-Sanchez MA, Liu J, Hannon GJ and Parker R: Control of translation and mRNA degradation by miRNAs and siRNAs. Genes Dev 20: 515-524, 2006.

29. Shimono Y, Zabala M, Cho RW, Lobo N, Dalerba P, Qian D, Diehn M, Liu H, Panula SP, Chiao E, et al: Downregulation of miRNA-200c links breast cancer stem cells with normal stem cells. Cell 138: 592-603, 2009.

30. Chen J, Chen Y and Chen Z: MiR-125a/b regulates the activation of cancer stem cells in paclitaxel-resistant colon cancer. Cancer Invest 31: 17-23, 2013.

31. Li L, Li Z, Kong X, Xie D, Jia Z, Jiang W, Cui J, Du Y, Wei D, Huang S, et al: Down-regulation of microRNA-494 via loss of SMAD4 increases FOXM1 and $\beta$-catenin signaling in pancreatic ductal adenocarcinoma cells. Gastroenterology 147: 485-497.e18, 2014.

32. Peng XH, Huang HR, Lu J, Liu X, Zhao FP, Zhang B, Lin SX, Wang L, Chen HH, Xu X, et al: MiR-124 suppresses tumor growth and metastasis by targeting Foxq1 in nasopharyngeal carcinoma. Mol Cancer 13: 186-198, 2014.

33. Zhang J, Yang Y, Yang T, Yuan S, Wang R, Pan Z, Yang Y, Huang G, Gu F, Jiang B, et al: Double-negative feedback loop between microRNA-422a and forkhead box (FOX)G1/Q1/E1 regulates hepatocellular carcinoma tumor growth and metastasis. Hepatology 61: 561-573, 2015.

34. Zhang Z, Ma J, Luan G, Kang L, Su Y, He Y and Luan F: MiR-506 suppresses tumor proliferation and invasion by targeting FOXQ1 in nasopharyngeal carcinoma. PLoS One 10: e0122851, 2015.

35. Xiang XJ, Deng J, Liu YW, Wan LY, Feng M, Chen J and Xiong JP: MiR-1271 inhibits cell proliferation, invasion and EMT in gastric cancer by targeting FOXQ1. Cell Physiol Biochem 36 $1382-1394,2015$

36. Ford SA and Blanck G: Signal persistence and amplification in cancer development and possible, related opportunities for novel therapies. Biochim Biophys Acta 1855: 18-23, 2015.

37. Nusse R and Varmus HE: Many tumors induced by the mouse mammary tumor virus contain a provirus integrated in the same region of the host genome. Cell 31: 99-109, 1982

38. Ochoa-Hernández AB, Juárez-Vázquez CI, Rosales-Reynoso MA and Barros-Núñez P: WNT- $\beta$-catenin signaling pathway and its relationship with cancer. Cir Cir 80: 389-398, 2012 (In Spanish).

39. Christensen J, Bentz S, Sengstag T, Shastri VP and Anderle P. FOXQ1, a novel target of the Wnt pathway and a new marker for activation of Wnt signaling in solid tumors. PLoS One 8: e60051, 2013.

40. Fan DM, Feng XS, Qi PW and Chen YW: Forkhead factor FOXQ1 promotes TGF- $\beta 1$ expression and induces epithelialmesenchymal transition. Mol Cell Biochem 397: 179-186, 2014.

41. Katsuno Y, Lamouille S and Derynck R: TGF- $\beta$ signaling and epithelial-mesenchymal transition in cancer progression. Curr Opin Oncol 25: 76-84, 2013.

42. Peng $X$, Luo Z, Kang Q, Deng D, Wang Q, Peng H, Wang S and Wei Z: FOXQ1 mediates the crosstalk between TGF- $\beta$ and Wnt signaling pathways in the progression of colorectal cancer. Cancer Biol Ther 16: 1099-1109, 2015.

43. Yap KL, Sysa-Shah P, Bolon B, Wu RC, Gao M, Herlinger AL, Wang F, Faiola F, Huso D, Gabrielson K, et al: Loss of NAC1 expression is associated with defective bony patterning in the murine vertebral axis. PLoS One 8: e69099, 2013

44. Mackler S, Pacchioni A, Degnan R, Homan Y, Conti AC, Kalivas $\mathrm{P}$ and Blendy JA: Requirement for the POZ/BTB protein $\mathrm{NAC1}$ in acute but not chronic psychomotor stimulant response. Behav Brain Res 187: 48-55, 2008

45. Shih Ie M, Nakayama K, Wu G, Nakayama N, Zhang J and Wang TL: Amplification of the ch19p13.2 NACC1 locus in ovarian high-grade serous carcinoma. Mod Pathol 24: 638-645, 2011.

46. Nakayama K, Nakayama N, Wang TL and Shih IeM: NAC-1 controls cell growth and survival by repressing transcription of Gadd45GIP1, a candidate tumor suppressor. Cancer Res 67: 8058-8064, 2007.

47. Gao M, Shih IeM and Wang TL: The role of forkhead box Q1 transcription factor in ovarian epithelial carcinomas. Int J Mol Sci 13: 13881-13893, 2012.

48. Hanahan D and Weinberg RA: Hallmarks of cancer: The next generation. Cell 144: 646-674, 2011
49. Bicknell KA: Forkhead (FOX) transcription factors and the cell cycle: Measurement of DNA binding by FoxO and FoxM transcription factors. Methods Mol Biol 296: 247-262, 2005.

50. Wonsey DR and Follettie MT: Loss of the forkhead transcription factor FoxM1 causes centrosome amplification and mitotic catastrophe. Cancer Res 65: 5181-5189, 2005.

51. Meng F, Speyer CL, Zhang B, Zhao Y, Chen W, Gorski DH, Miller FR and Wu G: PDGFR $\alpha$ and $\beta$ play critical roles in mediating Foxq1-driven breast cancer stemness and chemoresistance. Cancer Res 75: 584-593, 2015.

52. Ushkaryov YA, Petrenko AG, Geppert M and Südhof TC: Neurexins: Synaptic cell surface proteins related to the alphalatrotoxin receptor and laminin. Science 257: 50-56, 1992.

53. Rowen L, Young J, Birditt B, Kaur A, Madan A, Philipps DL, Qin S, Minx P, Wilson RK, Hood L, et al: Analysis of the human neurexin genes: Alternative splicing and the generation of protein diversity. Genomics 79: 587-597, 2002.

54. Tabuchi K and Südhof TC: Structure and evolution of neurexin genes: Insight into the mechanism of alternative splicing. Genomics 79: 849-859, 2002.

55. Kusinska R, Górniak P, Pastorczak A, Fendler W, Potemski P, Mlynarski W and Kordek R: Influence of genomic variation in FTO at $16 \mathrm{q} 12.2$, MC4R at $18 \mathrm{q} 22$ and NRXN3 at $14 \mathrm{q} 31$ genes on breast cancer risk. Mol Biol Rep 39: 2915-2919, 2012.

56. Sun HT, Cheng SX, Tu Y, Li XH and Zhang S: FoxQ1 promotes glioma cells proliferation and migration by regulating NRXN3 expression. PLoS One 8: e55693, 2013.

57. Harper JW, Adami GR, Wei N, Keyomarsi K and Elledge SJ: The p21 Cdk-interacting protein Cip1 is a potent inhibitor of G1 cyclin-dependent kinases. Cell 75: 805-816, 1993.

58. Dworakowska D, Jassem E, Jassem J, Boltze C, Wiedorn KH, Dworakowski R,Skokowski J,Jaśkiewicz K and CzestochowskaE: Absence of prognostic significance of $\mathrm{p} 21$ (WAF1/CIP1) protein expression in non-small cell lung cancer. Acta Oncol 44: 75-79, 2005.

59. Abbas T and Dutta A: p21 in cancer: Intricate networks and multiple activities. Nat Rev Cancer 9: 400-414, 2009.

60. Kaneda H, Arao T, Tanaka K, Tamura D, Aomatsu K, Kudo K, Sakai K, De Velasco MA, Matsumoto K, Fujita Y, et al: FOXQ1 is overexpressed in colorectal cancer and enhances tumorigenicity and tumor growth. Cancer Res 70: 2053-2063, 2010.

61. Folkman J: Tumor angiogenesis: Therapeutic implications. N Engl J Med 285: 1182-1186, 1971.

62. Folkman J: Angiogenesis in cancer, vascular, rheumatoid and other disease. Nat Med 1: 27-31, 1995.

63. Volpert OV, Dameron KM and Bouck N: Sequential development of an angiogenic phenotype by human fibroblasts progressing to tumorigenicity. Oncogene 14: 1495-1502, 1997.

64. Ellis LM and Hicklin DJ: VEGF-targeted therapy: Mechanisms of anti-tumour activity. Nat Rev Cancer 8: 579-591, 2008.

65. Klein CA: Parallel progression of primary tumours and metastases. Nat Rev Cancer 9: 302-312, 2009.

66. Williams SA, Anderson WC, Santaguida MT and Dylla SJ: Patient-derived xenografts, the cancer stem cell paradigm, and cancer pathobiology in the 21st century. Lab Invest 93: 970-982, 2013.

67. Timpl R, Rohde H, Robey PG, Rennard SI, Foidart JM and Martin GR: Laminin - a glycoprotein from basement membranes. J Biol Chem 254: 9933-9937, 1979.

68. Colognato $\mathrm{H}$ and Yurchenco PD: Form and function: The laminin family of heterotrimers. Dev Dyn 218: 213-234, 2000.

69. Ross JB, Huh D, Noble LB and Tavazoie SF: Identification of molecular determinants of primary and metastatic tumour re-initiation in breast cancer. Nat Cell Biol 17: 651-664, 2015.

70. Baeriswyl V and Christofori G: The angiogenic switch in carcinogenesis. Semin Cancer Biol 19: 329-337, 2009.

71. Bergers $G$ and Benjamin LE: Tumorigenesis and the angiogenic switch. Nat Rev Cancer 3: 401-410, 2003.

72. Stauffer JK, Scarzello AJ, Jiang Q and Wiltrout RH: Chronic inflammation, immune escape, and oncogenesis in the liver: A unique neighborhood for novel intersections. Hepatology 56: 1567-1574, 2012

73. Qian BZ and Pollard JW: Macrophage diversity enhances tumor progression and metastasis. Cell 141: 39-51, 2010.

74. Xia L, Huang W, Tian D, Zhang L, Qi X, Chen Z, Shang X, Nie Y and $\mathrm{Wu} \mathrm{K}$ : Forkhead box Q1 promotes hepatocellular carcinoma metastasis by transactivating ZEB2 and Versican V1 expression. Hepatology 59: 958-973, 2014. 
75. Larue L and Bellacosa A: Epithelial-mesenchymal transition in development and cancer: Role of phosphatidylinositol 3' kinase/ AKT pathways. Oncogene 24: 7443-7454, 2005.

76. Barouki $\mathrm{R}$ and Coumoul $\mathrm{X}$ : Cell migration and metastasis markers as targets of environmental pollutants and the Aryl hydrocarbon receptor. Cell Adhes Migr 4: 72-76, 2010.

77. Dietrich $\mathrm{C}$ and Kaina B: The aryl hydrocarbon receptor $(\mathrm{AhR})$ in the regulation of cell-cell contact and tumor growth. Carcinogenesis 31: 1319-1328, 2010.

78. Feng J, Zhang X, Zhu H, Wang X, Ni S and Huang J: FoxQ1 overexpression influences poor prognosis in non-small cell lung cancer, associates with the phenomenon of EMT. PLoS One 7: e39937, 2012.

79. Zhu Z, Zhu Z, Pang Z, Xing Y, Wan F, Lan D and Wang H: Short hairpin RNA targeting FOXQ1 inhibits invasion and metastasis via the reversal of epithelial-mesenchymal transition in bladder cancer. Int J Oncol 42: 1271-1278, 2013

80. Gao M, Wu RC, Herlinger AL, Yap K, Kim JW, Wang TL and Shih IeM: Identification of the NAC1-regulated genes in ovarian cancer. Am J Pathol 184: 133-140, 2014.

81. Sehrawat A, Kim SH, Vogt A and Singh SV: Suppression of FOXQ1 in benzyl isothiocyanate-mediated inhibition of epithelial-mesenchymal transition in human breast cancer cells. Carcinogenesis 34: 864-873, 2013.

82. Zhang H, Meng F, Liu G, Zhang B, Zhu J, Wu F, Ethier SP, Miller $F$ and Wu G: Forkhead transcription factor foxq1 promotes epithelial-mesenchymal transition and breast cancer metastasis. Cancer Res 71: 1292-1301, 2011.

83. Chambers AF, Groom AC and MacDonald IC: Dissemination and growth of cancer cells in metastatic sites. Nat Rev Cancer 2: 563-572, 2002.

84. Steeg PS: Tumor metastasis: Mechanistic insights and clinical challenges. Nat Med 12: 895-904, 2006.

85. Mengual L, Ars E, Lozano JJ, Burset M, Izquierdo L, IngelmoTorres M, Gaya JM, Algaba F, Villavicencio H, Ribal MJ, et al: Gene expression profiles in prostate cancer: Identification of candidate non-invasive diagnostic markers. Actas Urol Esp 38: 143-149, 2014.

86. Pei Y, Wang P, Liu H, He F and Ming L: FOXQ1 promotes esophageal cancer proliferation and metastasis by negatively modulating CDH1. Biomed Pharmacother 74: 89-94, 2015.

87. Huang W, Chen Z, Shang X, Tian D, Wang D, Wu K, Fan D and Xia L: Sox12, a direct target of FoxQ1, promotes hepatocellular carcinoma metastasis through up-regulating Twist1 and FGFBP1. Hepatology 61: 1920-1933, 2015.

88. Sánchez-Tilló E, Siles L, de Barrios O, Cuatrecasas M, Vaquero EC, Castells A and Postigo A: Expanding roles of ZEB factors in tumorigenesis and tumor progression. Am J Cancer Res 1: 897-912, 2011.
89. Xiong H, Hong J, Du W, Lin YW, Ren LL, Wang YC, Su WY, Wang JL, Cui Y, Wang ZH, et al: Roles of STAT3 and ZEB1 proteins in E-cadherin down-regulation and human colorectal cancer epithelial-mesenchymal transition. J Biol Chem 287: 5819-5832, 2012.

90. Cong N, Du P, Zhang A, Shen F, Su J, Pu P, Wang T, Zjang J, Kang C and Zhang Q: Downregulated microRNA-200a promotes EMT and tumor growth through the wnt/ $\beta$-catenin pathway by targeting the E-cadherin repressors ZEB1/ZEB2 in gastric adenocarcinoma. Oncol Rep 29: 1579-1587, 2013.

91. Kiefer JC: Back to basics: Sox genes. Dev Dyn 236: 2356-2366, 2007.

92. Kamachi Y and Kondoh H: Sox proteins: Regulators of cell fate specification and differentiation. Development 140: 4129-4144, 2013.

93. Hoser M, Potzner MR, Koch JM, Bösl MR, Wegner M and Sock E: Sox 12 deletion in the mouse reveals nonreciprocal redundancy with the related Sox4 and Sox 11 transcription factors. Mol Cell Biol 28: 4675-4687, 2008.

94. Jung HY and Yang J: Unraveling the TWIST between EMT and cancer stemness. Cell Stem Cell 16: 1-2, 2015.

95. Tassi E, Al-Attar A, Aigner A, Swift MR, McDonnell K, Karavanov A and Wellstein A: Enhancement of fibroblast growth factor (FGF) activity by an FGF-binding protein. J Biol Chem 276: 40247-40253, 2001.

96. Tassi E, McDonnell K, Gibby KA, Tilan JU, Kim SE, Kodack DP, Schmidt MO, Sharif GM, Wilcox CS, Welch WJ, et al: Impact of fibroblast growth factor-binding protein-1 expression on angiogenesis and wound healing. Am J Pathol 179: 2220-2232, 2011.

97. Qin J, Xu Y, Li X, Wu Y, Zhou J, Wang G and Chen L: Effects of lentiviral-mediated Foxp1 and Foxq1 RNAi on the hepatocarcinoma cell. Exp Mol Pathol 96: 1-8, 2014.

98.Palchaudhuri R and Hergenrother PJ: Transcript profiling and RNA interference as tools to identify small molecule mechanisms and therapeutic potential. ACS Chem Biol 6: 21-33, 2011

99. Uprichard SL: The therapeutic potential of RNA interference. FEBS Lett 579: 5996-6007, 2005.

100. Bao B, Azmi AS, Aboukameel A, Ahmad A, Bolling-Fischer A, Sethi S, Ali S, Li Y, Kong D, Banerjee S, et al: Pancreatic cancer stem-like cells display aggressive behavior mediated via activation of FoxQ1. J Biol Chem 289: 14520-14533, 2014.

101. Wattenberg LW: Inhibition of carcinogenic effects of polycyclic hydrocarbons by benzyl isothiocyanate and related compounds. J Natl Cancer Inst 58: 395-398, 1977. 